\title{
Workplace to support the teamwork in the early stages of the product development process
}

\section{Journal Article}

Author(s):

Kunz, Andreas (D); Kennel, Thomas; Mazzone, Andrea; Müller, Stephan

Publication date:

2004

Permanent link:

https://doi.org/10.3929/ethz-a-006039475

Rights / license:

In Copyright - Non-Commercial Use Permitted

Originally published in:

Mécanique \& Industries 5(2), https://doi.org/10.1051/meca:2004019 


\title{
Workplace to support the teamwork in the early stages of the product development process
}

\author{
Andreas M. Kunz ${ }^{a}$, Thomas Kennel, Andrea Mazzone and Stephan Müller \\ Swiss Federal Institute of Technology (ETH), Center for Product Development, ICVR - Innovation Center Virtual Reality, \\ Tannenstrasse 3, 8092 Zurich, Switzerland
}

Received 30 June 2003, Accepted 20 October 2003

\begin{abstract}
The teamwork characterizes the early stages of the product development process. The teamwork in these early stages is characterized by simultaneous writing and sketching. Since the work is done on paper a later use of the generated ideas with computer programs is hindered. This represents a break in the logical concatenation of the product development process and thus results in a loss of information. This implies that it is necessary to provide the group with a tool that collects the information already during writing and sketching. A technical workplace is described that uses techniques from virtual reality to allow a real teamwork in these early stages. Using picture acquisition and color separation techniques different pencils can be detected simultaneously in real time on a horizontal surface. Since only active parts are detected by the picture acquisition no quantization errors can occur caused by other objects on the surface like paper, elbow or hands. The realized teamwork space offers an A1-size work area onto which a simultaneous input is possible with four pens of different color. For the construction of the device a material has to be evaluated that is suitable for a back-projection.
\end{abstract}

Key words: Product development process / simultaneous work / virtual reality / picture acquisition / real time / technical workplace / color separation / teamwork / sketching / active pens

\section{Introduction}

Although in many stages of the product development process modern information technologies are already used and considerable successes are achieved, the early stages of the product development process are still carried out very traditionally [1]. Most of the teamwork is performed with traditional paper-based methods. Such procedures complicate the later use of the generated data for recording, presentation, or its use in future tasks. If the posters that are created during a team session will be needed again, they are photographed, scanned or digitally reentered in order to use them in a digital way. This represents a break in the logical concatenation of the product development process and thus results in a loss of information. A lot of information is only available in the so-called "meta-layer" and thus not written down explicitly, but is only available from the context on the generated poster.

Therefore teamwork is often understood as a presentation of ideas in front of a group and a subsequent discussion. However, very often the actual idea is already available at this moment and is only modified. For this kind of teamwork diverse devices are available: flipcharts,

\footnotetext{
${ }^{a}$ Corresponding author: kunz@imes.mavt.ethz.ch
}

whiteboards and chalkboards, as well as electronical touch-sensitive boards. All these media are characterized by the vertical orientation of the device and the working method is clearly defined for the presentation and moderation in front of a team.

Conceptual ideas and the basis for new products however, are created in smaller groups and with a disordered, parallel working method. Such work in so-called small working groups is not moderated, and few or no rules exist which arrange the cooperation within the team. The work is characterized by the horizontal work area for individual work or for the common sketching of new ideas. The participants of such a small group work in parallel on this common sketching area in order to achieve a common result. Since touch-sensitive work areas are designed for presentation purposes they are not suitable for this kind of teamwork. In addition, they accept only a serial input and thus a parallel working is not possible. Furthermore, it is required that the board is only touched with the pen's tip. This is only possible when the touch-sensitive board is in an upright position; if the board is horizontally orientated the surface is not only touched with the pen tip but also by hand. Quantisation errors occur because of this and the position of the pen's tip is not detected correctly. 

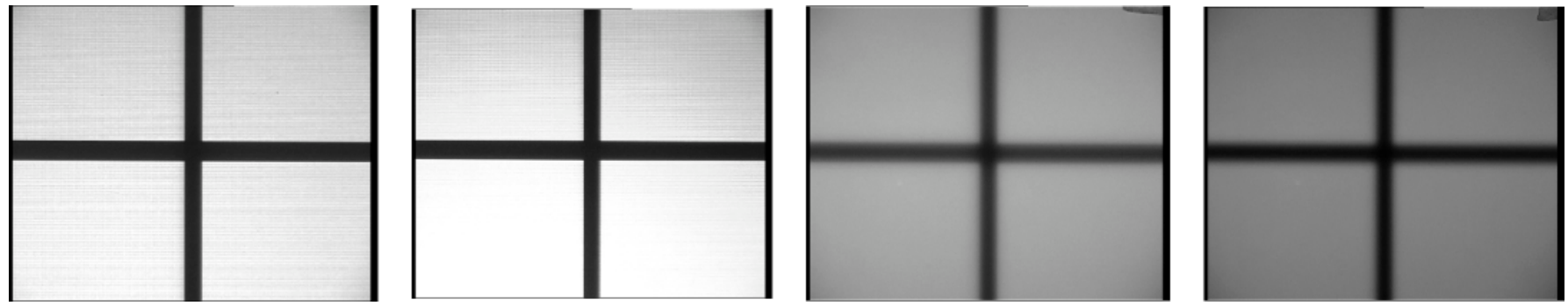

Fig. 1. Diffusion behaviour of the examined materials.

A further important criterion is the size of the available work area. If the work area is limited to a DIN A4 size there are already tools available that are based on an inductive principle. However, the size of these devices is not suitable for a work in smaller groups. It is inherent to all those devices that they do not support a simultaneous input with several pens, but rather only a serial input which is not typical for working in a small group [2].

The scope of this paper is to introduce a device for smaller groups that allows a simultaneous input on a large workspace and the reuse of generated ideas within later stages of development. The system should not hinder or influence the natural working method, but only support the later use of the generated data.

\section{Contributions}

The created teamwork place offers a DIN A1 size work area, which allows a simultaneous input with four pens of different colours. For the construction of the device a material was evaluated that is suitable for a back-projection. Beside this the material has to be insensitive to scratching and it has to be inflexible. The materials glass, Plexiglas and paper were examined. Plexiglas with $3 \mathrm{~mm}$ thickness and different tints of 010, 017, 060 and 072 was examined. In particular, the diffusion- and the hot spot behaviour were in the focus of interest [3].

\section{Investigation of the projection material}

For the investigation of the diffusion behaviour a test pattern was projected onto the material that shows a black cross on a white background. Figure 1 shows the different diffusion behaviours of the individual material.

As shown in Figure 1 only the tints 010 and 017 are suitable for a back projection. With higher tints the diffusion of the material is so strong that the projected picture becomes dark and blurred. The hotspot behaviour is also of interest. The hotspot is the visibility of the projector lens on the screen in cases of a back projection. In order to measure this hotspot the projector was placed 2.5 meters behind the screen so that the complete screen area was illuminated. The luminance was measured with a lux meter at different places on the screen. In this test only Perspex with the tints 010 and 017 was examined since the other material is not suitable for the projection. In order to reproduce real world conditions the viewing angle was changed as opposed to the distance of the lux meter. It was varied from 60 degrees upwards to 30 degrees downwards against the perpendicular in the vertical plane and 50 degrees to the left and to the right in the horizontal plane.

As it can be seen from Figure 2, Plexiglas 017 has a better distribution of brightness: the hotspot is considerably smaller. However, Plexiglas has the disadvantage that the projected picture can be recognized by the user only under a small viewing angle against perpendicular. If this angle increases, (i.e. if the user looks diagonally onto the projection screen) the pictures becomes too dark and the tool is no longer suitable for teamwork. Furthermore the flexibility of the Plexiglas is very disturbing during writing and sketching.

Therefore, other material combinations were examined. A combination of glass, white paper and translucent Plexiglas was used for the final preliminary model. This material combination has almost no hotspot but a higher attenuation of the transmitted light occurs. However, due to the high light intensity of the available beamers this drawback can be ignored. It is much more important that this material combination provides a larger angle of light radiation that guarantees good visibility of the projected image to the user, even under large viewing angles against the perpendicular.

The paper in combination with clear Plexiglas and glass shows a very diffuse radiation so that the projected picture can still be recognized under a large viewing angle against perpendicular without noticeable darkening or colour corruption of the image (Fig. 3). This is important because the users do not always stand orthogonally to the picture but see the picture on the screen under a certain angle. Figure 4 shows a front view as well as a diagonal view onto the projection screen under an angle of $45^{\circ}$ against perpendicular.

\section{Setup of the interaction table, the so-called "creatable"}

With this evaluated projection material the prototype of the device was built as shown in Figure 5 .

As shown in Figure 5 a colour camera and a computer are integrated into the table as well as a projector. 

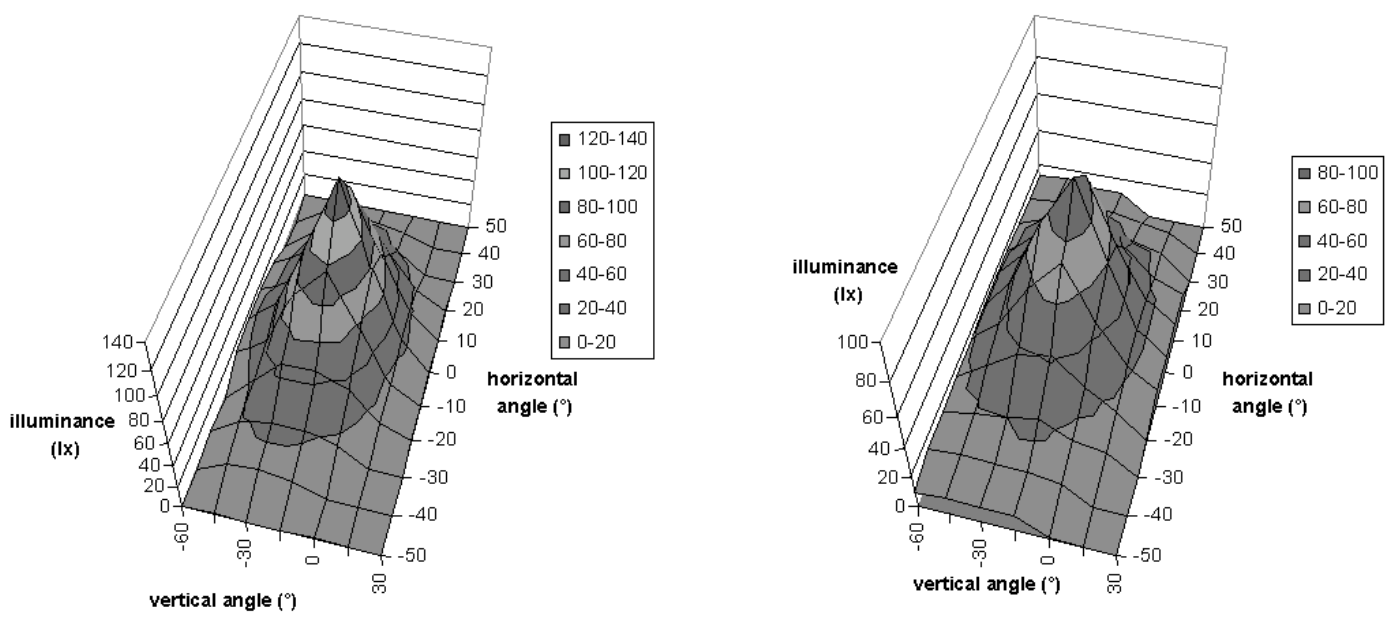

Fig. 2. Hot spot behaviour of the examined materials Perspex 010 and 017.

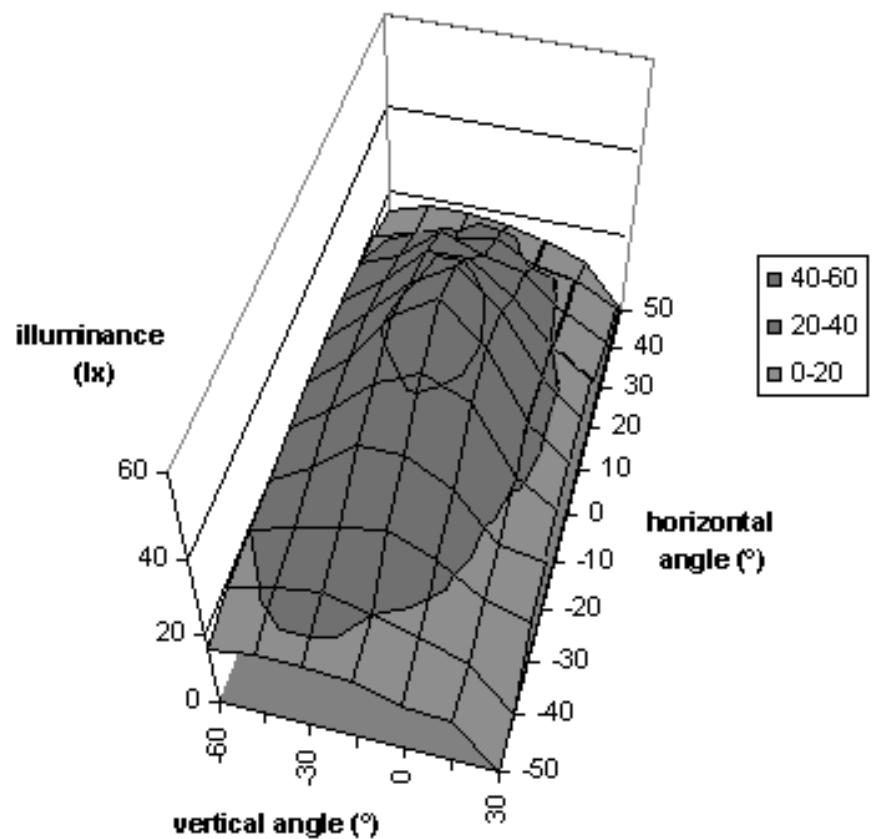

Fig. 3. Hot spot behaviour of the examined paper Utoplex 80.

In order to achieve the required size of the projected image on the screen with a given angle of aperture of the projector lens the beam is redirected once by a mirror. The camera has a direct view onto the projection screen. The light spot from the pen is registered by the camera and digitised by the computer. This signal is used by an application (e.g. a sketching tool) as a mouse information that is assigned to the light pen. The digitised image elements are back-projected by the a projector onto the work area again so that a realistic write impression arises [4].

Figure 6 shows the realized preliminary model, which was carried out as a desk variant. On a $600 \times 900 \mathrm{~mm}$ large surface a simultaneous writing and sketching of up to four users is possible. Every point on the projection screen is within the reach from every user's position. The base of the desk is smaller than its top to offer sufficient space is available for the feet and it is possible to stand close to the desk.

\section{Design of the light pen}

An ultrabright light-emitting diode (13000 mcd) was integrated into a pen in order to use it for writing and sketching on the table's surface. By integrating a microswitch into the pen it is only activated when its tip is slightly pressed against the surface of the screen. This avoids that a light spot is detected above the table's surface, which would result in a faulty interpretation of writing and sketching. In order to realize a maximally realistic write feeling, the light-emitting diode is integrated into a felt as it is also used for standard pens. The pen has the following basic construction (see Fig. 7).

A battery, a microswitch, a resistor and a LED are integrated into an original housing of a pen in order to improve the natural feeling of writing.

\section{Segmentation software and tip recognition}

A SONY DFW-500 CCD-camera with progressivescan technology and a YUV-output is used for the picture acquisition. The camera is connected to the framegrabber card via firewire. The architecture of the $\mathrm{C}++$ software [5] that is needed for the colour segmentation and thus for the recognition of the light pen is shown in the following flowchart (Fig. 8).

In a first step the YUV-colour values coming from the camera are splitted up into RGB-colour values using the following formula (1):

$$
\begin{aligned}
& {\left[\begin{array}{l}
R \\
G \\
B
\end{array}\right]=1.164+(Y-16)} \\
& +\left[\begin{array}{c}
1.596 \cdot(U-128) \\
-0.813 \cdot(U-128) \\
0
\end{array}\right]+\left[\begin{array}{c}
0 \\
-0.391 \cdot(V-128) \\
2.018 \cdot(V-128)
\end{array}\right]
\end{aligned}
$$



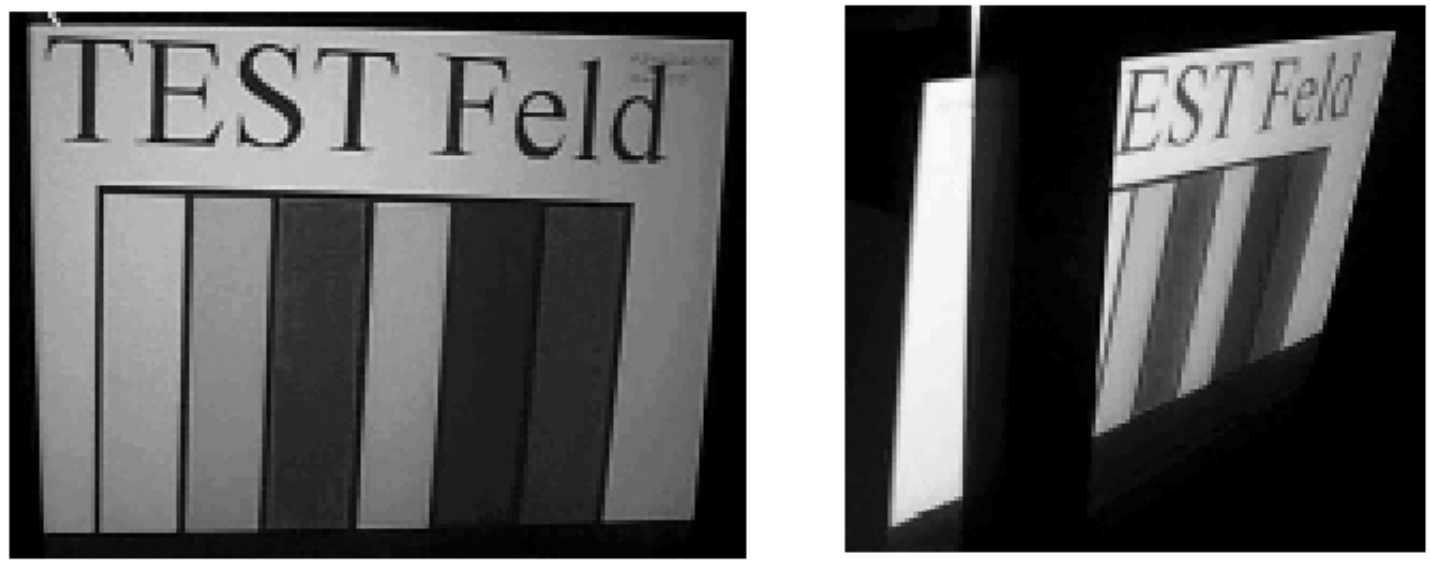

Fig. 4. Diffuse reflectivity of the used material.

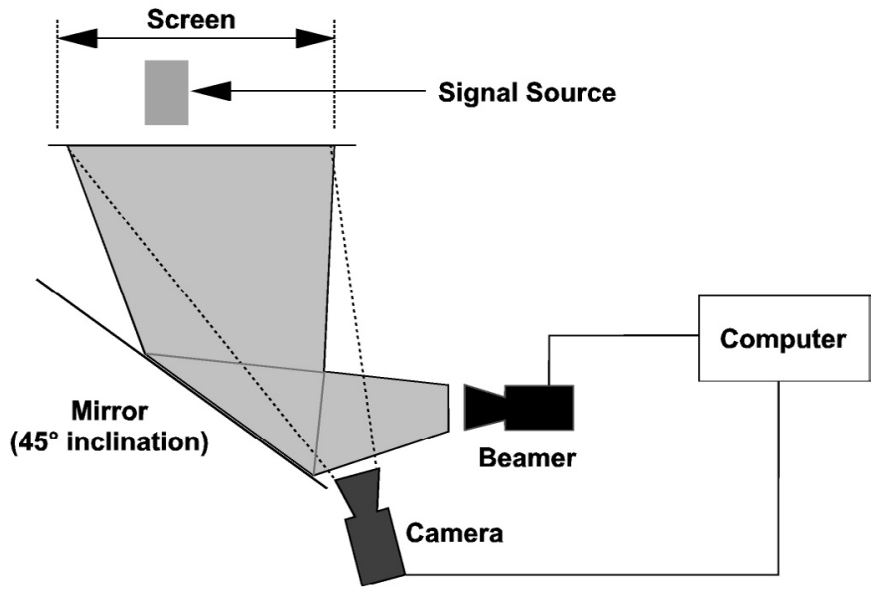

Fig. 5. Constructive design of the teamwork place.

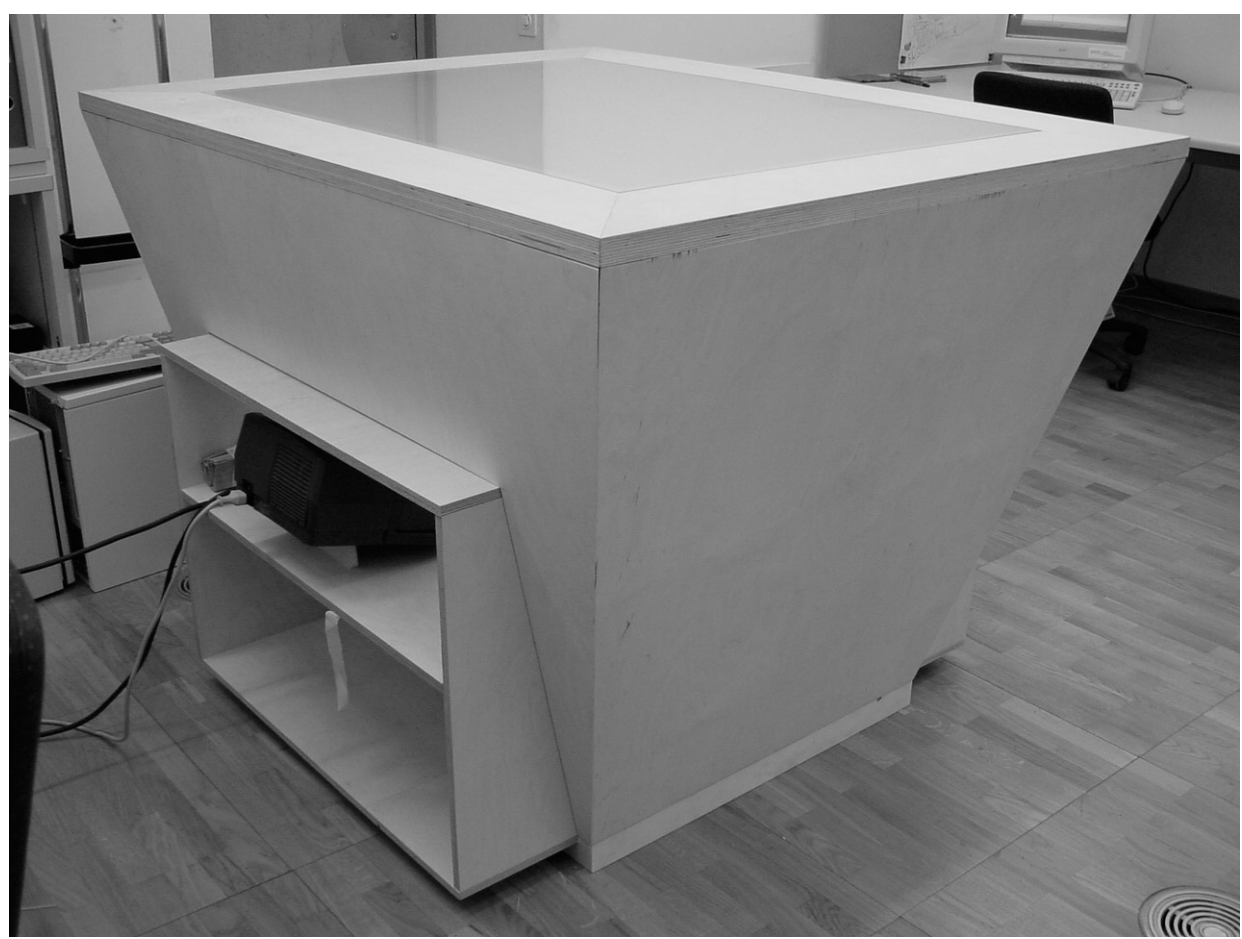

Fig. 6. Realized preliminary model. 

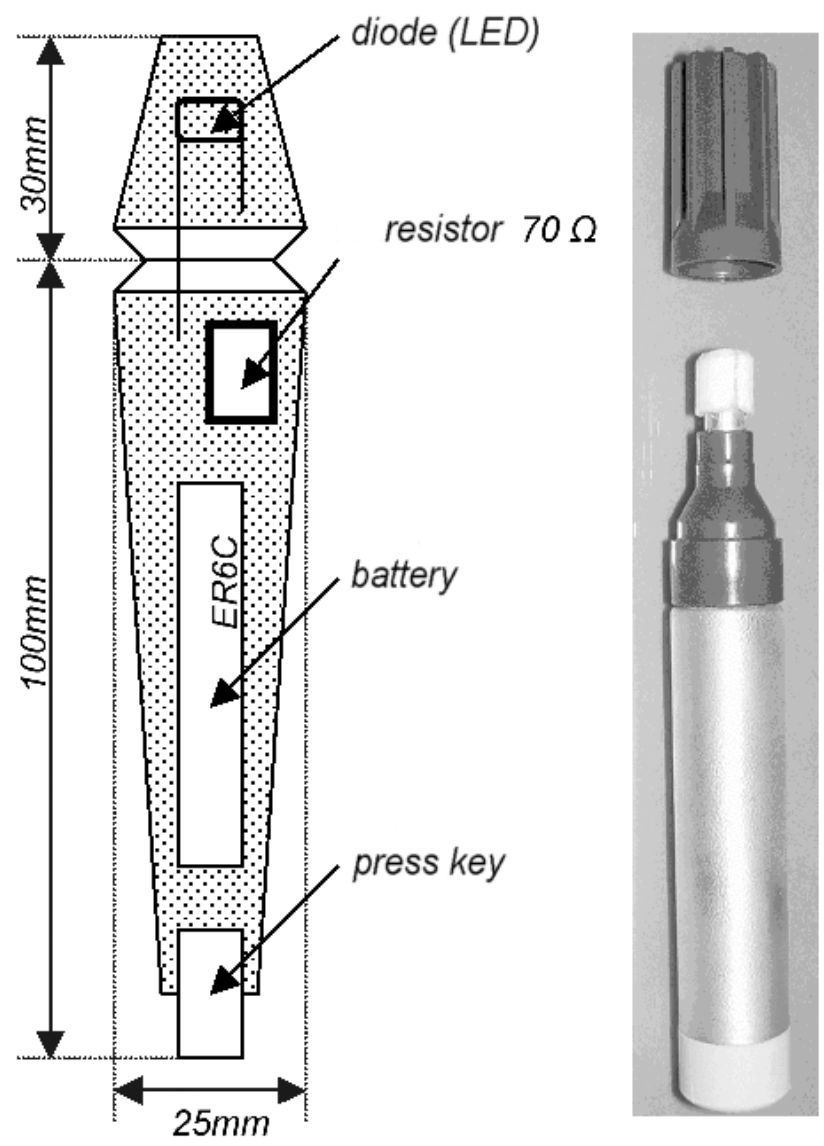

Fig. 7. Construction of the pen.

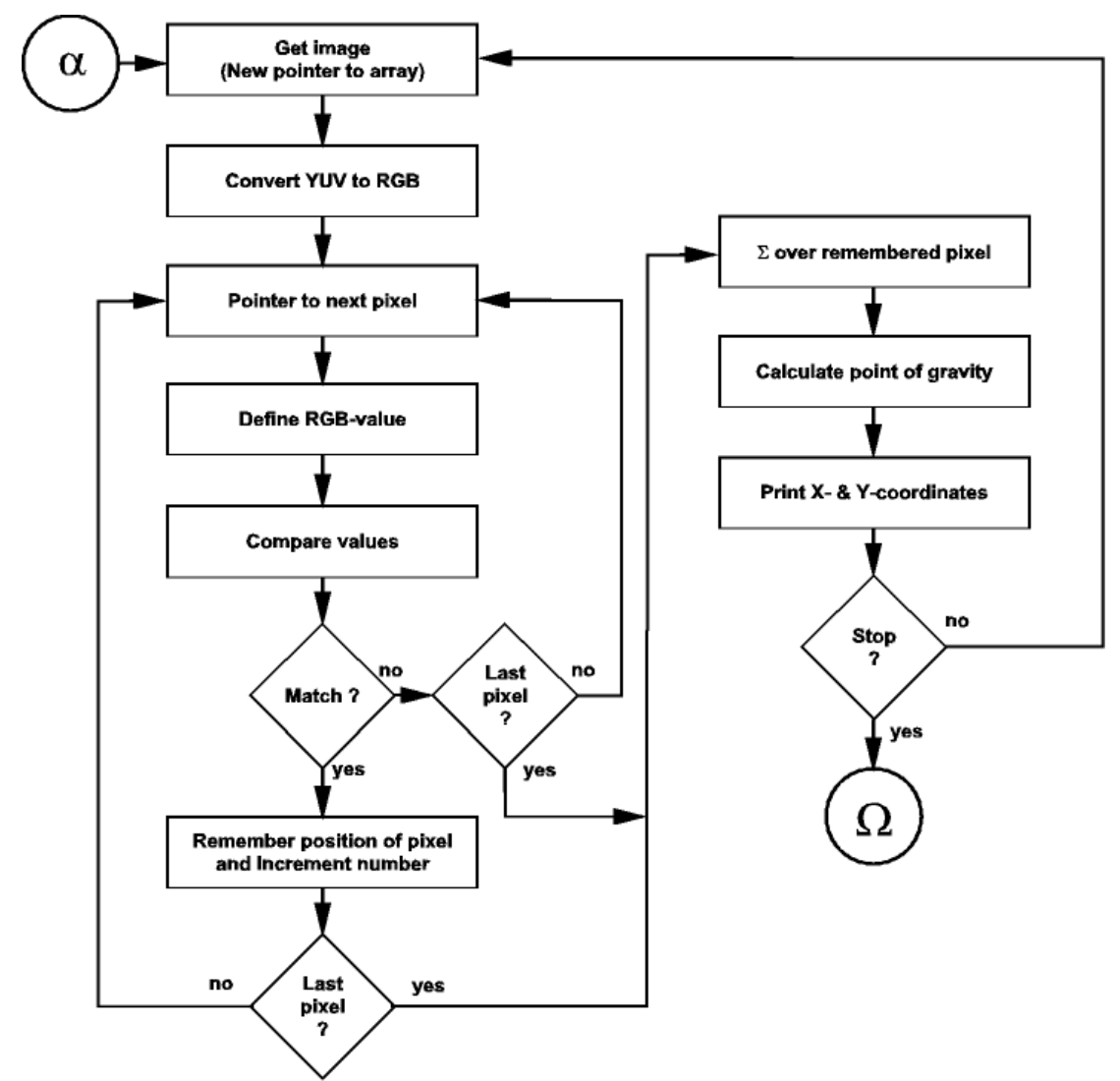

Fig. 8. Flowchart of the implemented software. 
In a further step the software must be able to balance deviations in the colour values between the ones emitted from the diode and the RGB-colour values coming from the camera. This is particularly necessary when additional external influences occur to the system like daylight or artificial light.

Every emitted and/or measured pixel is described in the RGB colour field by a vector as follows (2):

$$
\left\{\begin{array}{l}
R_{\text {is }} \\
G_{\text {is }} \\
B_{\text {is }}
\end{array}\right\} \text { resp. }\left\{\begin{array}{l}
R_{\text {des }} \\
G_{\text {des }} \\
B_{\text {des }}
\end{array}\right\}
$$

Now a maximum distance $D$ can be defined between the two pixels in the colour field (see Fig. 9). If the distance between two pixels in the colour room is smaller than a default maximum it is assumed that both pixels are of the same colour. The distance of two pixels in the threedimensional room can be calculated as follows (3):

$$
D=\sqrt{\left(R_{\mathrm{des}}-R_{\mathrm{is}}\right)^{2}+\left(G_{\mathrm{des}}-G_{\mathrm{is}}\right)^{2}+\left(B_{\mathrm{des}}-B_{\mathrm{is}}\right)^{2}}
$$

In a next step the centre of the pixel cloud is calculated that was measured during the image processing. Thus, an unambiguous assignment to one point becomes possible. This calculation of the centre is simplified in order to shorten the CPU time and the overall latency time of the system. For each possible colour of a diode two arrays are defined that are used for the $x$ - and the $y$-coordinates of a diode. Since only writing and sketching is supported, the $z$-axis is not needed. With every recognized pixel the corresponding coordinates are written into these arrays and a counter is increased by 1 . In order to calculate the centre of a pixel cloud the addresses in the arrays are summed up and are divided by the number of coordinates. From that the $x$ - and $y$-position of each diode can be obtained.

\section{Test interface}

For the testing of the functions a user interface was realized that allows a graphical output of the camera signal. The position of the recognized diodes is continuously updated as a numerical output of the calculated coordinates. The interface is shown in Figure 10.

\section{Conclusions}

The setup of an electronic teamwork place was introduced, which allows parallel writing and sketching inputs during a team session. Specific materials were evaluated that allow a back projection combined with a small hot spot behaviour. Furthermore these materials must have a diffuse radiation behaviour so that the projected image is recognizable even under a large angle perpendicular to the screen. Perspex in specific tints has a good hot spot behaviour, but the scattering angle is very small so that

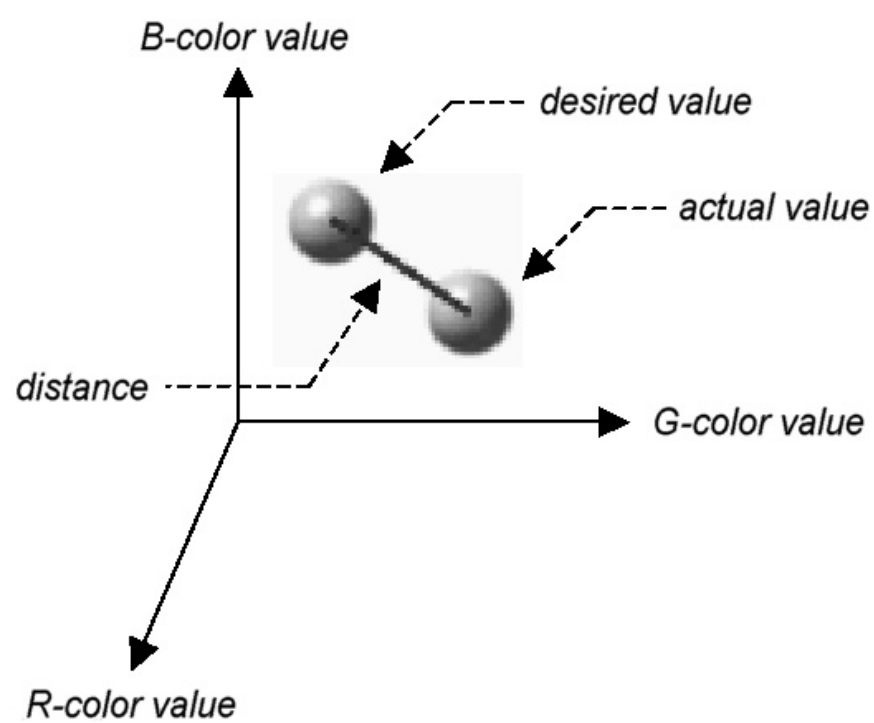

Fig. 9. RGB color field with actual value and desired value.

it could not be used here. Instead a material combination of glass and paper was chosen. This allows a recognition of the projected image under a big angle against the orthogonal of the screen.

With the selected projection materials a small teamwork place and a back projection onto a work area of $600 \times 900 \mathrm{~mm}$ was realized. Specific pens with integrated light-emitting diodes were build that are recognized by a camera beneath the projection screen and a digitalized picture can be acquired.

The basic construction of the evaluating-software was introduced that allows the recognition and assignment of the image elements and thus the pens. In particular, the analysis of the picture has to be as fast as possible in order to keep the latency time very small. A simple evaluatingsoftware allows first tests about the processing speed of the entire system.

\section{Future work}

Future works will deal with the possibility how the speed of the picture recognition and picture evaluation can be increased. Furthermore, the software for the innovation process and the generation of ideas will be designed in such a way that it will not hinder the above processes. Thus, it is guaranteed that the actual innovation process is not influenced disturbingly but is effectively supported by the electronic working place.

Acknowledgements. The above work was made possible within the KTI-Investigation project "Innopro" that examines the technical support of the early stages of the innovation process. We would like to thank all persons participated in this project. 


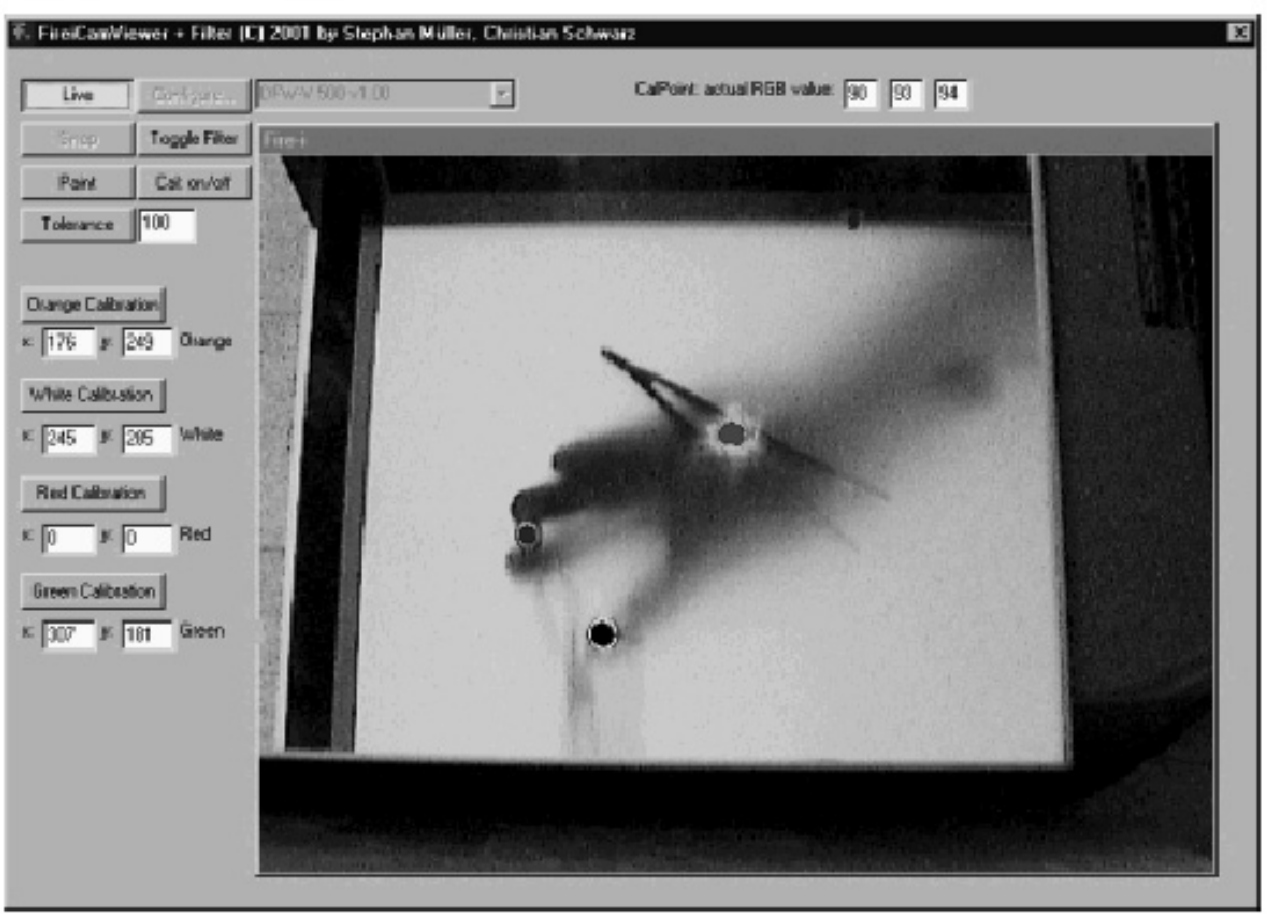

Fig. 10. Realized interface.

\section{References}

[1] E. Arias, H. Eden, G. Fischer, A. Gorman, E. Scharff, Creating Shared Understanding through Collaborative Deisgn, CHI-Proceedings, University of Colorado, Boulder

[2] T.P. Moran, P. Chiu, W. van Melle, G. Kurtenbach, Implicit Structures for Pen-Based Systems Within a Freeform Interaction Paradigm, Proceedings of CHI 1995
[3] H. Hermann, Untersuchung neuartiger Projektionswerkstoffe, Studienarbeit ETH Zurich, 2001

[4] J.A. Brotherton, J.R. Bhalodia, G.D. Abowd, Automated Capture, Integration, and Visualization of Multiple Media Streams, Proceedings IEEE 1998

[5] D.J. Kruglinski, S. Wingo, G. Shepherd, Programming Microsoft Visual $\mathrm{C}++$ 5th Edition, Microsoft Press, Redmont, Washington, USA, 1998, ISBN 1-57231-857-0 